\title{
A Simple, Novel Approach to Capsulorhexis Formation in the Setting of A Mature Cataract and Miotic Pupil
}

This article was published in the following Dove Press journal: Clinical Ophthalmology

\author{
Stephen A LoBue $\mathbb{D}^{1,2}$ \\ Prashant Tailor ${ }^{3}$ \\ Thomas D LoBue (iD) \\ 'Department of Ophthalmology, LoBue \\ Laser and Eye Medical Center Inc, \\ Murrieta, CA, USA; ${ }^{2}$ Lincoln Medical \\ Center, Affiliated to Weill Cornell \\ Medical College, Bronx, NY, USA; \\ ${ }^{3}$ Medical College of Georgia, Augusta, \\ GA, USA
}

Purpose: To report a simple, effective technique for surgeons creating a capsulorhexis in patients with pupillary miosis and a dense, mature cataract.

Methods: A single center, two-year retrospective chart review examined 1408 phacoemulsification cataract surgeries. The criteria for inclusion involved a dense, mature cataract and a pupil that was unresponsive to pharmacologic dilation preoperatively. A standardized technique was used for all cases consisting of a $1 \mathrm{~mm}$ corneal paracentesis and $2.4 \mathrm{~mm}$ temporal clear corneal incision. Synecholysis was performed if present, followed by the insertion of a $6.25 \mathrm{~mm}$ malyugin ring under cohesive viscoelastic. The cohesive viscoelastic was removed via the irrigation aspiration tip. The paracentesis was sealed with a small amount of viscoelastic and an air bubble was placed in the anterior chamber. The anterior capsule was then painted with trypan blue. The air bubble and trypan blue were then replaced by a dispersive viscoelastic. Curvolinear capsulorrhexis was performed followed by standard phacoemulsion.

Results: Nine patients ranging from $76 \pm 12$ years (mean \pm standard deviation) met the criteria with a $4+$ NS $(n=5)$, white mature $(n=3)$, or deep brunescent $(n=1)$ cataract and $3 \mathrm{~mm}$ pupil preoperatively. Pupillary miosis was caused by posterior synechia in $44.5 \%$ of the cases followed by pharmacologic interactions from tamsulosin and donepezil in $22.25 \%$ of cases respectively. One case involved idiopathic miosis likely from aging. Capsulorhexis formation was successful in all cases with no capsular tear, vitreous loss, or conversion to extracapsular cataract extraction (ECCE). However, one case had cortex retention requiring a second procedure for removal.

Conclusion: Dense, mature cataracts and small pupils both compromise the view for the surgeon and may be significant risk factors for training ophthalmologists. Thus, good visualization of the anterior capsule and peripheral nucleus with our simple technique facilitates a reliable capsulorhexis, potentially limiting the risk of complications for ophthalmic surgeons.

Keywords: dense cataract, miosis, IFIS, dementia, novice surgeon, pupil ring
Correspondence: Thomas D LoBue LoBue Laser and Eye Medical Center Inc, 40700 California Oaks Road, Ste 106, Murrieta, CA 92562, USA

$\mathrm{Tel}+1$ 951-696-II35

Email Thomas.lobue@lobueeyecenters.com

\section{Introduction}

Cataracts account for one of the leading causes of preventable blindness across the world. As a result, cataract surgery is a fundamental skill learned during ophthalmology residency. However, the art of cataract surgery has vastly evolved over the past few decades, especially in developed countries. Techniques have shifted from intracapsular cataract extraction (ICCE) and extracapsular cataract extraction (ECCE) to phacoemulsification. Nevertheless, the transition to phacoemulsification from ECCE has been associated with an increase in complications for training 
ophthalmologists. ${ }^{1,2}$ Complications, such as anterior capsule tears, posterior capsular rupture (PCR) with or without vitreous loss, zonular dialysis/dehiscence, and nuclear fragment loss, are often attributed to inadequate continuous curvilinear capsulorhexis (CCC) or poor visualization of the peripheral nucleus during phacoemulsification. ${ }^{2}$ The increase in intraocular complications is likely due to a steep learning curve as complications such as vitreous loss have demonstrated to significantly decrease after the first 80 cases in training residents. ${ }^{3}$

Even with greater experience, certain patient demographics predispose young surgeons to increased complications. Patient-related risk factors often include dementia, anxiety, advanced age ${ }^{4}$ as well as individuals with deep-set eyes, prominent brows, and limited corneal visibility. However, some of the greatest intraocular risks for novice surgeons involve a miotic pupil, ${ }^{5}$ dense nuclear sclerosis, ${ }^{6-8}$ zonular pathology (antecedent trauma or pseudoexfoliation), ${ }^{6,9}$ and prior pars plana vitrectomy. ${ }^{10}$

However, the combination of pupillary miosis and dense cataracts may even be more problematic for novice surgeons as the view of the capsule and nucleus is significantly obscured, thus limiting the ability to create an adequate $\mathrm{CCC}$ without complication. Therefore, we developed a standardized approach with our simple technique which may facilitate the creation of a capsulorhexis in patients with pupillary miosis and dense nuclear sclerosis.

\section{Methods}

A total of 1408 phacoemulsification cataract surgeries were examined at a single ambulatory surgical center at LoBue Laser and Eye Medical Center Inc. The retrospective study was conducted in accordance with the Declaration of Helsinki for human subjects in research. Individual consent was obtained for each subject who met the criteria for inclusion with all data anonymized to maintain confidentiality. Inclusion criteria involved patients with $4+$ nuclear sclerosis (NS), white mature, or brunescent cataracts along with impaired preoperative pupillary dilation classified as $3 \mathrm{~mm}$ or less. Patients were excluded if only one of the above criteria were met. All patients included in the study were operated by a single surgeon using a standardized technique described below. Phacoemulsification was performed with the Alcon Centurion (Fort Worth, TX).

A (superior on the right eye, inferior on the left) $1 \mathrm{~mm}$ corneal paracentesis was created (Figure 1A). Methylparaben free xylocaine was injected intracamerally to numb the iris.

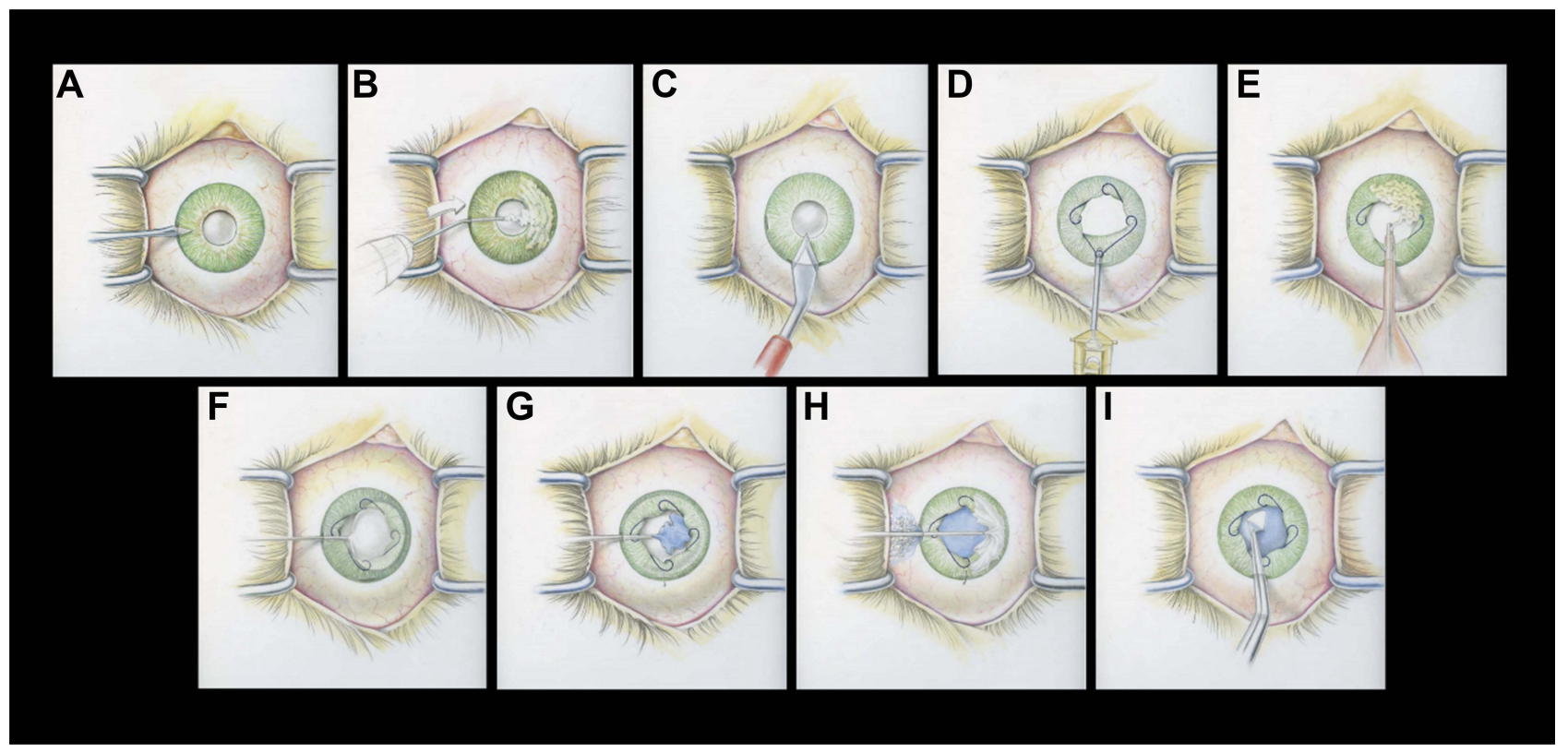

Figure I The right eye from the surgeon's perspective depicting pupillary miosis and a dense, mature cataract. A standardized technique was used to create a continuous curvilinear capsulorhexis (CCC). (A): Imm corneal paracentesis. (B): Methylparaben free xylocaine was injected intracamerally to numb the iris then cohesive viscoelastic was used to fill the anterior chamber. (C): $2.4 \mathrm{~mm}$ temporal clear corneal incision with a microkeratome blade. Posterior synechiae, if present, were broken with a collar button. (D): A $6.25 \mathrm{~mm}$ malyugin ring was placed to expand the pupil. (E): Cohesive viscoelastic was then removed via an irrigating/aspiration tip. (F): A small amount of viscoelastic was then used to seal the paracentesis. A 27-gauge cannula was then used to inject air through the paracentesis. If air was leaking through the temporal corneal wound, a temporary single 10-0 nylon could be placed in the incision. $(\mathbf{G})$ : Trypan blue was then injected through the paracentesis to stain the anterior capsule. $(\mathbf{H})$ : The air bubble and trypan blue were subsequently removed by filling the anterior chamber with a dispersive viscoelastic through the paracentesis. (I): The I0-0 nylon suture was removed from the temporal incision if it was previously placed. A $\sim 5 \mathrm{~mm}$ CCC was initiated with a cystotome and completed using Utrata Forceps. 
Cohesive viscoelastic was then used to fill the anterior chamber (Figure 1B). Next, a 2.4mm temporal clear corneal incision was made with a microkeratome blade (Figure 1C). Posterior synechiae, if present, was broken with a collar button. Subsequently, a $6.25 \mathrm{~mm}$ malyugin ring was placed to expand the pupil (Figure 1D). Cohesive viscoelastic was then removed via an irrigating/aspiration tip, with careful attention to not damage the anterior capsule (Figure 1E). A small amount of viscoelastic was then used to seal the paracentesis. Next a 27gauge cannula was then used to inject air through the paracentesis (Figure 1F). If air was leaking through the corneal wound, a temporary single $10-0$ nylon could be placed in the temporal incision. Trypan blue was then injected through the paracentesis to stain the anterior capsule (Figure 1G). The air bubble and trypan blue were subsequently removed by filling the anterior chamber with a dispersive viscoelastic through the paracentesis (Figure 1H). Once again, importance was placed on visualizing the cannula within the air bubble so that the integrity of the anterior capsule was not compromised. The 10-0 nylon suture was removed from the temporal incision if it was previously placed. Finally, a $\sim 5 \mathrm{~mm}$ continuous curvilinear capsulorhexis was initiated with a cystotome and completed using Utrata Forceps (Figure 1I).

\section{Results}

Nine patients ranging from $76 \pm 12$ years (mean \pm standard deviation) met the criteria with a $4+\mathrm{NS}(\mathrm{n}=5)$, white mature $(n=3)$, or deep brunescent $(n=1)$ cataract and $3 \mathrm{~mm}$ pupil preoperatively (Table 1 ). Five out of the nine patients were female with preoperative best corrective visual acuity (BCVA) for distance ranging from 20/70 to hand motion vision. Postoperative visual acuity improved in all patients, ranging from 20/20 to $20 / 70$ (Table 2). Visual acuity was $20 / 40$ or better in $56 \%$ of the patients. Cataract surgery was uneventful in all nine patients. Capsulorhexis formation was successful in all cases with no capsular tear, vitreous loss, or conversion to ECCE. However, one case had cortex retention requiring removal (Table 2).

The past medical and eye history of the cohort was analyzed. Common conditions included glaucoma with either a trabeculectomy or Ahmed valve $(n=2)$, severe keratitis with corneal transplant $(\mathrm{n}=1)$, severe uveitis secondary to HIV ( $\mathrm{n}=1)$, benign prostatic hypertrophy $(\mathrm{n}=2)$, Alzheimer's dementia $(\mathrm{n}=2)$, proliferative diabetic retinopathy with vitrectomy $(\mathrm{n}=1)$, and hypothyroidism $(\mathrm{n}=1$, Table 1$)$. Pupillary miosis was caused by posterior synechia in $44.5 \%$ of the cases followed by pharmacologic interactions from
Table I Patient Demographics, History, and Cataract Grade

\begin{tabular}{|l|l|l|l|}
\hline Age & Gender & $\begin{array}{l}\text { Past Medical/Eye } \\
\text { History }\end{array}$ & Cataract Grade \\
\hline 9 I & M & BPH & White mature \\
\hline 78 & F & Glaucoma s/p Ahmed valve & White mature \\
\hline 73 & F & $\begin{array}{l}\text { Severe keratitis s/P corneal } \\
\text { transplant }\end{array}$ & White mature \\
\hline 54 & M & Severe uveitis from HIV & $4+$ NS \\
\hline 87 & M & BPH & $4+$ NS \\
\hline 7 I & F & $\begin{array}{l}\text { PDR, vitrectomy, } \\
\text { Alzheimer's }\end{array}$ & $\begin{array}{l}4+\text { NS, 3+ CS, 2+ } \\
\text { PSC }\end{array}$ \\
\hline 87 & M & Glaucoma s/P trab & $4+$ NS \\
\hline 74 & F & Alzheimer's & $\begin{array}{l}\text { 4+ NS, 3+ CS, I+ } \\
\text { PSC }\end{array}$ \\
\hline 68 & F & $\begin{array}{l}\text { Hyperthyroidism } \\
\text { brunescence }\end{array}$ \\
\hline
\end{tabular}

Abbreviations: PDR, proliferative diabetic retinopathy; Trab, trabeculectomy; S/P, status post; BPH, benign prostatic hypertrophy; NS, nuclear sclerosis; CS, cortical spoking; PSC, poster subcapsular cataract.

Table 2 Surgical Outcomes and Mechanism for Miosis

\begin{tabular}{|l|l|l|l|}
\hline $\begin{array}{l}\text { Preop } \\
\text { VA }\end{array}$ & $\begin{array}{l}\text { Postop } \\
\text { VA }\end{array}$ & Complications & $\begin{array}{l}\text { Mechanism for } \\
\text { Miosis }\end{array}$ \\
\hline HM & $20 / 40$ & N/A & Tamsulosin \\
CF & $20 / 30$ & N/A & Posterior synechia \\
HM & $20 / 50$ & N/A & Posterior synechia \\
$20 / 300$ & $20 / 70$ & N/A & Posterior synechia \\
$20 / 80$ & $20 / 40$ & N/A & Tamsulosin \\
$20 / 300$ & $20 / 50$ & N/A & Donepezil \\
$20 / 70$ & $20 / 40$ & Retained Cortex & Posterior synechia \\
$20 / 80$ & $20 / 25$ & N/A & Donepezil \\
$20 / 400$ & $20 / 20$ & N/A & Idiopathic \\
\hline
\end{tabular}

Abbreviations: Preop, preoperative; Postop, postoperative; VA, visual acuity.

tamsulosin and donepezil in $22.25 \%$ of cases respectively. One case involved idiopathic pupillary miosis likely secondary to iris sphincter sclerosis from aging.

\section{Discussion}

Cataract extraction is one of the most common intraocular procedures in medicine. Although common, cataract extraction is a very technical surgery, where each phase of the procedure is fundamentally based on the success of the previous step. Since the transition from ECCE to phacoemulsification, one of the most critical aspects of cataract extraction involves the formation of the CCC. 
However, new surgeons often perceive CCC or phacoemulsification as one of the most challenging techniques to initially master. ${ }^{1,2}$ Yet, these technical procedures become significantly more difficult when the view of the capsule becomes obscured due to dense nuclear sclerosis ${ }^{6-8}$ or miosis. ${ }^{5}$ Impaired peripheral capsule visibility increases the risk of PCR which may result in vitreous loss, dislocated intraocular lenses, glaucoma, uveitis, cystoid macular edema, and retinal detachment. ${ }^{11}$ In order to improve capsular visibility while minimizing complications, we developed a simple, standardized technique which facilitates the creation of a capsulorhexis and aids in phacoemulsification in patients with pupillary miosis and dense cataracts.

As mentioned above, small pupils are a well-known risk factor for posterior capsule tears, retained lens fragments, and vitreous loss during phacoemulsification. The minimum threshold for successful capsulorhexis and phacoemulsification typically involves pupil diameters of 4.5-5.0 $\mathrm{mm}$ for experience surgeons ${ }^{12}$ and $6 \mathrm{~mm}$ for novice surgeons. ${ }^{5}$ The etiology of pupillary miosis is vast but can range from sphincter sclerosis from aging, diabetes mellitus, pseudoexfoliation, Intraoperative Floppy Iris Syndrome (IFIS), cholinergic agonists, surgical trauma, synechia, uveitis, glaucoma, and miosis following femtosecond capsulotomy.

In our cohort, $44.5 \%$ of the patients developed miosis due to posterior synechia secondary to previous surgery, uveitis, or glaucoma. Typically, adhesions between the iris and the anterior lens capsule can be efficiently lysed with a spatula or a collar button as demonstrated in our technique. We favor a collar button in order to minimize damage to surrounding structures during synechiolysis. On the other hand, $44.5 \%$ of patients developed pharmacologic induced miosis from either tamsulosin or donepezil. Acetylcholinesterase inhibitors such as donepezil are first-line symptomatic treatments for Alzheimer's dementia which function by inhibiting degradation of acetylcholine in the synaptic clefts, thereby improving cholinergic neurotransmission. Estermann et al found that mean pupil diameter significantly decreases after four weeks of oral treatment of donepezil. ${ }^{13}$ Yet, the most important facet to recognize is that dementia is a strong independent risk factor for intraoperative complications during phacoemulsification. ${ }^{10}$ Patients with even mild dementia may become disoriented, agitated, and uncooperative once placed under a drape, leading to excessive head movements. In our cohort, we had two patients affected by dementia. In patients with high levels of anxiety or known history of dementia, we stagger single intravenous push sedation in order to keep patients calm and comfortable. Typically, $1 \mathrm{mg}$ Versed is given immediately prior to moving the patient into the operating room (OR). Once in the OR, $25 \mathrm{mcg}$ of fentanyl is subsequently given during time out. Then as the surgeon is scrubbing for surgery, $10 \mathrm{mg}$ of ketamine is delivered to the patient. Overall, ketamine is a great addition for anxious, demented patients as it controls pain and eye movement without affecting intraocular pressure. ${ }^{14}$ We also find in our practice that patients generally enjoy the ketamine sedation and report an overall positive experience with minimal adverse reactions.

Nonetheless, the pharmacological effects of tamsulosin are associated with more concerning effects, even for experienced surgeons. Alpha-1A antagonists, such as tamsulosin, have been linked with a clinical triad including fluttering or billowing of the iris stroma, propensity for iris prolapse, and progressive pupillary constriction known as IFIS. ${ }^{15,16}$ This triad significantly increases the complexity of cataract surgery and risk for intraoperative complications. Since complications may be as high as $12.5 \%$, ${ }^{15}$ several methods have been developed to mitigate these risks. Hashemi et al detail techniques ranging from intracameral buffered lidocaine and epinephrine (e.g., EpiShugarcaine), ${ }^{17}$ viscomydriasis by combining cohesive and dispersive ophthalmic viscosurgical device (OVD), ${ }^{18}$ iris hooks or retractors, and expanding pupillary rings. ${ }^{16,19}$

Our technique incorporates both pupillary rings and two types of OVD, which is effective in facilitating mydriasis and stabilization of the iris. Cohesive viscoelastic is inserted through the paracentesis which temporarily deepens the anterior chamber, widens the pupil, and supports the iris. Next, a malyugin ring is inserted through the self-sealing, triplaner cornea wound, docking on the nasal and superior or inferior iris. All patients in our study had poor pupil dilation not exceeding $3 \mathrm{~mm}$ which improved to $\sim 6.25 \mathrm{~mm}$ after insertion of the ring. Poor preoperative pupil dilation is a predictive test for the severity of IFIS. $^{20}$ Thus, even with patients demonstrating attributes of severe IFIS, we experienced no intraoperative complications. The growing understanding of IFIS and its management techniques has led to a dramatic decrease in complications, as low as 0 to $0.6 \%$ for PCR and vitreous loss. $^{21,22}$ Using expanding pupillary rings, such as our technique, is highly effective in stabilizing the iris and improving the field of view of miotic conditions such as IFIS. ${ }^{16,19,21,23}$ Although iris retractors may also have a role in improving the field of view, ${ }^{19}$ the process is more 
invasive, ${ }^{19,24}$ tedious, and requires strategic placement in conditions of IFIS. ${ }^{25}$

The view of the surgeon is also significantly affected by the grade of the cataract. In our retrospective study, all patients had dense nuclear sclerosis which ranged from $4+$ NS, white mature, or brunescent cataracts. The red reflex was absent in all cases, limiting our view of the anterior and posterior capsule. Without an adequate view of the capsule, CCC becomes significantly more difficult, increasing the risk for radial tears toward or beyond the lens equator, vitreous loss, nuclear drop, and intraocular lens displacement. Previous studies among experienced surgeons (without capsular dye) have demonstrated that denser nuclear cataracts, paralleled increasing PCR rates from $0.7 \%$ to $16.7 \% .^{2}$ Capsulorhexis failure were also significantly affected by nuclear density, reaching $36.7 \%$ with the densest cataracts. ${ }^{2}$

A common approach to improve anterior capsular visibility in dense cataracts often incorporates intracapsular dye such as trypan blue. However, there are several methods in which the dye can be delivered, ranging from under air, ${ }^{26}$ under OVD, ${ }^{27}$ OVD + BSS, mixing with OVD, ${ }^{28}$ or one step intracameral injection. Although the safety and effectiveness of trypan blue under OVD versus air are similar, ${ }^{29}$ there are select advantages to each method. Thus, we developed a staining technique which incorporates both air and OVD.

In our technique, the paracentesis is sealed with a small amount of dispersive viscoelastic. The wound, if necessary, is sealed with a single 10-0 nylon suture before air is injected through a 27 gauge cannula through the paracentesis. In some instances, multiple air bubbles form in the anterior chamber which quickly coalesce into a single bubble after a few seconds. Trypan blue is then injected from the paracentesis, making sure to visualize the cannula in the air bubble. Our approach is advantageous for several reasons. For one, the anterior segment is well sealed, minimizing air leak and shallowing of the anterior chamber during capsular staining. Rapid changes of the lens-iris plane can lead to unintentional damage of the anterior capsule, compromising capsulorhexis formation. Secondly, staining under air prevents direct contact of the dye with the corneal endothelium and allows for better enhancement of the peripheral anterior capsule rim. As a result of better visualization, the rate of successful capsulorhexis formation significantly improves. The success rate for CCC for mature or hypermature cataracts may be as high as a $100 \%{ }^{30}$ as in our study when a capsular dye is used. The uniform staining of the anterior capsule with trypan may even be helpful for all grades of cataracts, especially in training ophthalmologists. ${ }^{31}$

After successful staining of the anterior capsule, dispersive viscoelastic is used to evacuate dye and air from the anterior chamber. Dispersive viscoelastic is a vital part of our technique because it helps maintain the anterior chamber depth while also protecting the endothelium. A shallow, crowded anterior chamber increases the incidence of iris prolapse and will shift the posterior capsule closer to the phaco tip, thus increasing the risk for PCR. However, in some cases, OVD may obscure the stained anterior capsule interface. This pitfall can be avoided by injecting a small amount of trypan, such as 1cc, while creating a brush stroke motion with the cannula. Nevertheless, once the anterior capsule is punctured using a cystotome blade, the stark contrast between the stained capsule and cataract makes the leading edge of the capsulorhexis clearly visible.

Overall, there are several limitations to our study. Due to our selective inclusion criteria, our population size is small and may not accurately reflect the true complication rates. Also, all cataract surgery was performed by a single, experienced surgeon. A prospective study, including a broader range of surgeon experience, may be needed to validate our findings.

\section{Conclusion}

Dense, mature cataracts and small pupils both compromise the view for the surgeon and may be significant risk factors for PCR in training ophthalmologists. In order to improve capsular visibility while minimizing complications, we developed a simple, standardized technique which facilitates the creation of a capsulorhexis in patients with pupillary miosis and dense cataracts. The technique incorporates a pupil expansile ring and capsular staining under both air and dispersive viscoelastic. Our method may also be beneficial for experienced surgeons performing cataract surgery with patients exhibiting several highrisk factors such as white mature/deep brunescent cataracts and miosis associated with IFIS or a history of dementia. IFIS has been associated with a high rate of complications, which has significantly been mitigated with better identification of high-risk patients and presurgical planning. Our technique, using a Malyugin ring along with cohesive OVD, stabilizes the iris and provides consistent dilation throughout the course of the case, minimizing complications in our study. Lastly, dementia is a strong independent risk factor for intraoperative complications which may be 
exacerbated in longer, more complex cases. We feel our technique along with an additional single, low dose IV sedation of ketamine, fentanyl, and versed are beneficial in creating a calmer, more cooperative patient.

\section{Acknowledgments}

The abstract was presented at the Association for Research in Vision and Ophthalmology (ARVO) 2019 in Vancouver.

\section{Disclosure}

The authors report no conflicts of interest int this work.

\section{References}

1. Dooley IJ, O'Brien PD. Subjective difficulty of each stage of phacoemulsification cataract surgery performed by basic surgical trainees. J Cataract Refract Surg. 2006;32(4):604-608. doi:10.1016/ j.jcrs.2006.01.045

2. Martin KR, Burton RL. The phacoemulsification learning curve: per-operative complications in the first 3000 cases of an experienced surgeon. Eye (Lond). 2000;14(Pt 2):190-195. doi:10.1038/eye.2000.52

3. Randleman JB, Wolfe JD, Woodward M, Lynn MJ, Cherwek DH, Srivastava SK. The resident surgeon phacoemulsification learning curve. Arch Ophthalmol. 2007;125(9):1215-1219. doi:10.1001/ archopht.125.9.1215

4. Jaycock P, Johnston RL, Taylor H, et al. The Cataract National Dataset electronic multi-centre audit of 55,567 operations: updating benchmark standards of care in the United Kingdom and internationally. Eye (Lond). 2009;23(1):38-49. doi:10.1038/sj.eye.6703015

5. Kim JY, Ali R, Cremers SL, Yun S-C, Henderson BA. Incidence of intraoperative complications in cataract surgery performed by left-handed residents. $J$ Cataract Refract Surg. 2009;35 (6):1019-1025. doi:10.1016/j.jcrs.2009.01.025

6. Rutar T, Porco TC, Naseri A. Risk factors for intraoperative complications in resident-performed phacoemulsification surgery. Ophthalmology. 2009;116(3):431-436. doi:10.1016/j.ophtha.2008.10.028

7. Briszi A, Prahs P, Hillenkamp J, Helbig H, Herrmann W. Complication rate and risk factors for intraoperative complications in resident-performed phacoemulsification surgery. Graefes Arch Clin Exp Ophthalmol. 2012;250(9):1315-1320. doi:10.1007/s00417-012-2003-y

8. Prakash G, Jhanji V, Sharma N, Gupta K, Titiyal JS, Vajpayee RB. Assessment of perceived difficulties by residents in performing routine steps in phacoemulsification surgery and in managing complications. Can J Ophthalmol. 2009;44(3):284-287. doi:10.3129/109-051

9. Chen M, Lamattina KC, Patrianakos T, Dwarakanathan S. Complication rate of posterior capsule rupture with vitreous loss during phacoemulsification at a Hawaiian cataract surgical center: a clinical audit. Clin Ophthalmol. 2014;8:375-378. doi:10.2147/OPTH.S57736

10. Blomquist PH, Morales ME, Tong L, Ahn C. Risk factors for vitreous complications in resident-performed phacoemulsification surgery. $J$ Cataract Refract Surg. 2012;38(2):208-214. doi:10.1016/j. jers.2011.10.001

11. Johansson B, Lundström M, Montan P, Stenevi U, Behndig A. Capsule complication during cataract surgery: long-term outcomes: Swedish Capsule Rupture Study Group report 3. J Cataract Refract Surg. 2009;35(10):1694-1698. doi:10.1016/j.jcrs.2009.05.027

12. Malyugin B. Cataract surgery in small pupils. Indian J Ophthalmol. 2017;65(12):1323-1328. doi:10.4103/ijo.IJO_800_17
13. Estermann S, Daepp G-C, Cattapan-Ludewig K, Berkhoff M, Frueh BE, Goldblum D. Effect of oral donepezil on intraocular pressure in normotensive Alzheimer patients. $J$ Ocul Pharmacol Ther. 2006;22(1):62-67. doi:10.1089/jop.2006.22.62

14. Cugini U, Lanzetta P, Nadbath P, Menchini U. Sedation with ketamine during cataract surgery. J Cataract Refractive Surg. 1997;23 (5):784-786. doi:10.1016/S0886-3350(97)80291-X

15. Chang DF, Campbell JR. Intraoperative floppy iris syndrome associated with tamsulosin. J Cataract Refract Surg. 2005;31 (4):664-673. doi:10.1016/j.jcrs.2005.02.027

16. Hashemi H, Seyedian MA, Mohammadpour M. Small pupil and cataract surgery. Curr Opin Ophthalmol. 2015;26(1):3-9. doi:10.1097/ ICU.0000000000000116

17. Shugar JK. Use of epinephrine for IFIS prophylaxis. $J$ Cataract Refract Surg. 2006;32(7):1074-1075. doi:10.1016/j.jcrs.2006.01.110

18. Arshinoff SA. Modified SST-USST for tamsulosin-associated intraoperative [corrected] floppy-iris syndrome. J Cataract Refract Surg. 2006;32(4):559-561. doi:10.1016/j.jcrs.2006.01.001

19. Malyugin BE. Recent advances in small pupil cataract surgery. Curr Opin Ophthalmol. 2018;29(1):40-47. doi:10.1097/ICU.0000000000000443

20. Casuccio A, Cillino G, Pavone C, Spitale E, Cillino S. Pharmacologic pupil dilation as a predictive test for the risk for intraoperative floppy-iris syndrome. $J$ Cataract Refract Surg. 2011;37 (8):1447-1454. doi:10.1016/j.jcrs.2011.02.030

21. Chang DF, Osher RH, Wang L, Koch DD. Prospective multicenter evaluation of cataract surgery in patients taking tamsulosin (Flomax). Ophthalmology. 2007;114(5):957-964. doi:10.1016/j. ophtha.2007.01.011

22. Neff KD, Sandoval HP, Fernández de Castro LE, Nowacki AS, Vroman DT, Solomon KD. Factors associated with intraoperative floppy iris syndrome. Ophthalmology. 2009;116(4):658-663. doi:10.1016/j.ophtha.2008.12.026

23. Chang DF. Use of Malyugin pupil expansion device for intraoperative floppy-iris syndrome: results in 30 consecutive cases. $J$ Cataract Refract Surg. 2008;34(5):835-841. doi:10.1016/j.jcrs.2008.01.026

24. Masket $\mathrm{S}$. Avoiding complications associated with iris retractor use in small pupil cataract extraction. J Cataract Refract Surg. 1996;22 (2):168-171. doi:10.1016/S0886-3350(96)80213-6

25. Oetting TA, Omphroy LC. Modified technique using flexible iris retractors in clear corneal cataract surgery. J Cataract Refract Surg. 2002;28(4):596-598. doi:10.1016/S0886-3350(01)01100-2

26. Melles GR, de Waard PW, Pameyer JH, Houdijn Beekhuis W. Trypan blue capsule staining to visualize the capsulorhexis in cataract surgery. J Cataract Refract Surg. 1999;25(1):7-9. doi:10.1016/ S0886-3350(99)80004-2

27. Khokhar S, Pangtey MS, Panda A, Sethi HS. Painting technique for staining the anterior lens capsule. J Cataract Refract Surg. 2003;29 (3):435-436. doi:10.1016/S0886-3350(02)01607-3

28. Dada VK, Sudan R, Sharma N, Dada T. Trypan blue with a viscoelastic agent. $J$ Cataract Refract Surg. 2002;28(2):205-206. doi:10.1016/s0886-3350(01)01337-2

29. Wong VWY, Lai TYY, Lee GKY, Lam PTH, Lam DSC. A prospective study on trypan blue capsule staining under air vs under viscoelastic. Eye (Lond). 2006;20(7):820-825. doi:10.1038/sj. eye. 6702032

30. Kothari K, Jain SS, Shah NJ. Anterior capsular staining with trypan blue for capsulorhexis in mature and hypermature cataracts. A preliminary study. Indian J Ophthalmol. 2001;49(3):177-180.

31. Dada T, Ray M, Bhartiya P, Vajpayee RB. Trypan-blue-assisted capsulorhexis for trainee phacoemulsification surgeons. $J$ Cataract Refract Surg. 2002;28(4):575-576. doi:10.1016/S0886-3350(02) 01316-0 


\section{Publish your work in this journal}

Clinical Ophthalmology is an international, peer-reviewed journal covering all subspecialties within ophthalmology. Key topics include: Optometry; Visual science; Pharmacology and drug therapy in eye diseases; Basic Sciences; Primary and Secondary eye care; Patient Safety and Quality of Care Improvements. This journal is indexed on PubMed
Central and CAS, and is the official journal of The Society of Clinical Ophthalmology (SCO). The manuscript management system is completely online and includes a very quick and fair peer-review system, which is all easy to use. Visit http://www.dovepress.com/ testimonials.php to read real quotes from published authors. 